\title{
Long-term Effectiveness of Trabectome (Ab-interno Trabeculectomy) Surgery
}

\author{
${ }^{1}$ Rick E Bendel, ${ }^{2}$ Michael T Patterson
}

\begin{abstract}
Aim: To evaluate the long-term safety and efficacy of ab-interno trabeculectomy with trabectome for the treatment of glaucoma.

Materials and methods: Data collected for 339 eyes which included demographics, intraocular pressure (IOP) measurements using Goldmann applanation tonometry, best-corrected visual acuity (BCVA), visual field results, optic nerve status, gonioscopic findings, prior glaucoma procedures, number of glaucoma medications and pain level. The main data points of interest were preoperative IOP vs. postoperative IOP and BCVA, medication use, pain status, and complications.
\end{abstract}

Results: Of the 339 eyes that underwent trabectome, we found a statistically significant reduction in IOP $(p<0.01)$ at final follow-up (average $=18.35$ months) of nearly $23 \%$, with a complication rate of $5.86 \%$. Furthermore, this reduction was maintained up to 8 years post procedure. LogMAR visual acuity was significantly improved in $69 \%$ of eyes at the final visit $(p<0.05)$, while only $1.77 \%$ of cases saw a significant reduction. Based on these findings, we determined a success rate of around $80 \%$ to 100 months following trabectome.

Conclusion: Trabectome is a safe and effective long term for most forms and severities of glaucoma.

Keywords: Intraocular pressure, Minimally invasive glaucoma surgery, Trabectome, Trabeculectomy, Retrospective chart review.

How to cite this article: Bendel RE, Patterson MT. Long-term Effectiveness of Trabectome (Ab-interno Trabeculectomy) Surgery. J Curr Glaucoma Pract 2018;12(3):119-124.

Source of support: Nil

Conflict of interest: None

\section{INTRODUCTION}

Glaucoma is the second leading cause of irreversible blindness worldwide. ${ }^{1,2}$ Current proven surgical techniques to treat glaucoma only slow the progression of optic nerve damage by reducing intraocular pressure, rather than reversing the damage. These surgeries typically require either an implant or filtration style procedure. However, implant methods are invasive and classic filtration

\footnotetext{
${ }^{1}$ Physician, ${ }^{2}$ Clinical Research Fellow

1,2Department of Ophthalmology, Mayo Clinic Foundation of Florida, Jacksonville, Florida, USA

Corresponding Author: Rick E Bendel, Physician, Department of Ophthalmology, Mayo Clinic Foundation of Florida, Jacksonville, Florida, USA, email: Bendel.Rick@mayo.edu
}

methods, such as trabeculectomy, result in a high com-

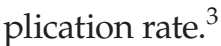

Ab-interno Trabeculectomy with Trabectome (Neomedix Corp) remains a promising, minimally invasive glaucoma procedure that decreases IOP by carefully ablating and removing some of the Trabecular meshwork and exposing collector channels, which facilitates aqueous humor outflow. ${ }^{4,5}$ The Trabectome is a surgical tool that was approved for use by the US Food and Drug Administration in $2004,{ }^{6}$ and gained popularity for the treatment of various forms of glaucoma. ${ }^{7}$ The Trabectome procedure presents a viable alternative to traditional trabeculectomy because of the absence of an external filtering bleb. Bleb failure and scarring remains one of the most prevalent complications associated with filtration surgeries and bleb maintenance requires lifelong follow-up postoperatively.

Many studies highlight the short-term IOP lowering ability, and low complication rate of trabectome, ${ }^{8-11}$ however, long-term data on its effectiveness and patient satisfaction remains scarce. In this retrospective chart study, we evaluate the long-term efficacy and safety of trabectome in patients with various types of glaucoma.

\section{MATERIALS AND METHODS}

A case series of subjects who had undergone ab-interno trabeculectomy with the trabectome from March of 2008 to June of 2016 was analyzed with the approval of the institutional review board and by the Declaration of Helsinki and the Health Insurance Portability and Accountability Act. All patients were previously diagnosed with glaucoma, and a single surgeon performed all procedures at the Mayo Clinic of Florida in Jacksonville.

Preoperative data was collected for 339 eyes which included demographics, IOP measurements using Goldmann Applanation Tonometry, BCVA, visual field results, optic nerve status, gonioscopic findings, prior glaucoma procedures, and many glaucoma medications. Postoperative data was collected for the following parameters: IOP, BVCA, complication status following an ocular exam, glaucoma medications and pain level using a verbal analog scale where patients were asked to gauge their pain as either no pain, mild pain, moderate pain or severe pain. Demographic data are summarized in Table 1. 
Table 1: Patient demographics and ocular status of all eyes that underwent trabectome

\begin{tabular}{|c|c|}
\hline Patient demographics & Percentage (\%) \\
\hline Number of eyes & 339 \\
\hline \multicolumn{2}{|l|}{ Age } \\
\hline Mean age & 75.5 \\
\hline Range & $36-93$ \\
\hline \multicolumn{2}{|l|}{ Sex } \\
\hline Male & 109 \\
\hline Female & 92 \\
\hline \multicolumn{2}{|l|}{ Race } \\
\hline Caucasian & 279 \\
\hline African American & 33 \\
\hline Asian & 8 \\
\hline Other & 19 \\
\hline \multicolumn{2}{|l|}{ Glaucoma type } \\
\hline POAG & 206 \\
\hline Pseudoexfoliation & 20 \\
\hline Pigmentary & 9 \\
\hline Narrow-angle (NAG) & 52 \\
\hline Trauma & 2 \\
\hline Unspecified & 43 \\
\hline Low tension & 4 \\
\hline \multicolumn{2}{|l|}{ Visual acuity } \\
\hline \multicolumn{2}{|l|}{ Average preoperative } \\
\hline \multicolumn{2}{|l|}{ Visual field } \\
\hline Mild & $20 / 40.8$ \\
\hline Moderate & 201 \\
\hline Severe & 98 \\
\hline \multicolumn{2}{|l|}{ Cup to disk } \\
\hline$<0.5$ & 40 \\
\hline $0.5-0.69$ & 31 \\
\hline $0.7-0.79$ & 115 \\
\hline $0.8-0.89$ & 75 \\
\hline$>0.9$ & 72 \\
\hline \multicolumn{2}{|l|}{ Prior surgery } \\
\hline LTP & 46 \\
\hline Trabeculectomy & 107 \\
\hline $\mathrm{PI}$ & 10 \\
\hline AVG & 28 \\
\hline
\end{tabular}

Angle: Shaffer grade

$0 \quad 10$

1115

$2 \quad 79$

3445

$4 \quad 60$

140

The main data points of interest were preoperative IOP vs. postoperative IOP, medication use, pain status, and complications. The average follow-up time was 18 months (range 3-100 months). Patients were diagnosed with the following types of glaucoma: pseudoexfoliation, primary open angle, narrow-angle, trauma, low tension, pigmentary, and other secondary forms of glaucoma (Table 1). The inclusion criteria were: the presence of uncontrolled glaucoma on maximum tolerated glaucoma medications.
For patients who had a preoperative IOP $>15$, success was defined as a postoperative IOP between 5 to $20 \mathrm{~mm} \mathrm{Hg}$, no increase in the number of glaucoma medications and no reduction of visual acuity. For patients who had a preoperative IOP $<15$, success was defined as an increase in visual acuity, no increase in IOP and no increase the number of glaucoma medications. Failure was a result of not meeting the success parameters or occurrence of prolonged pain and/or complications. Statistical analysis of the difference in IOP and number of glaucoma medications before and after surgery were carried out using a paired t-test where a $p$ value of $<0.05$ was considered statistically significant. A Kaplan-Meier analysis was employed to determine survival rates using our success definition to compensate for the varying follow up times. All statistical analyses were performed using GraphPad Prism.

In the operating room, brimonidine, antibiotic, and topical anesthetic drops were instilled in the operative eye. Then, the eye was draped using standard sterile technique. A temporal corneal incision was made, and lidocaine was injected into the anterior chamber (AC). Trabectome ablation of approximately 120 to 160 degrees of the trabecular meshwork was performed under gonioscopy using the NeoMedix Trabectome Goniolens. The patient's head was tilted 30 to 40 degrees away from the surgeon, and the microscope was also tilted 30 to 40 degrees toward the surgeon during each procedure. When combined with cataract removal and lens implant (IOL), the trabectome was always performed first. Any blood reflux was cleared from the AC. Carbachol was then injected into the AC, and the incisions were sealed with BSS. Dexamethasone was then injected subconjunctivally, and antibiotic drops were reapplied. When synechial closure was present, this area of the trabecular meshwork was avoided. The patient was positioned to the greatest extent possible to avoid synechial areas. During preoperative evaluation, if more than 1 clock hour of synechial closure was present, trabectome was not performed.

\section{RESULTS}

A total of 339 eyes were enrolled in this study that had been previously diagnosed with various forms of glaucoma. Mean IOP before undergoing trabectome was $18.01 \pm 1.2 \mathrm{~mm} \mathrm{Hg}$ and was significantly reduced to $13.89 \pm 1.5 \mathrm{mmHg}$ at the final follow-up visit $(p<0.01)$. The average reduction in IOP at the final follow-up was $19.62 \%$. Graph 1A compares preoperative IOP to posoperative measurements at various follow-up times. To assess the ability of eyes to maintain a long term stable IOP after undergoing trabectome, we looked specifically at patients who had follow-up data of 3,5 and 8 years, and found each cohort had a statically significant reduction 

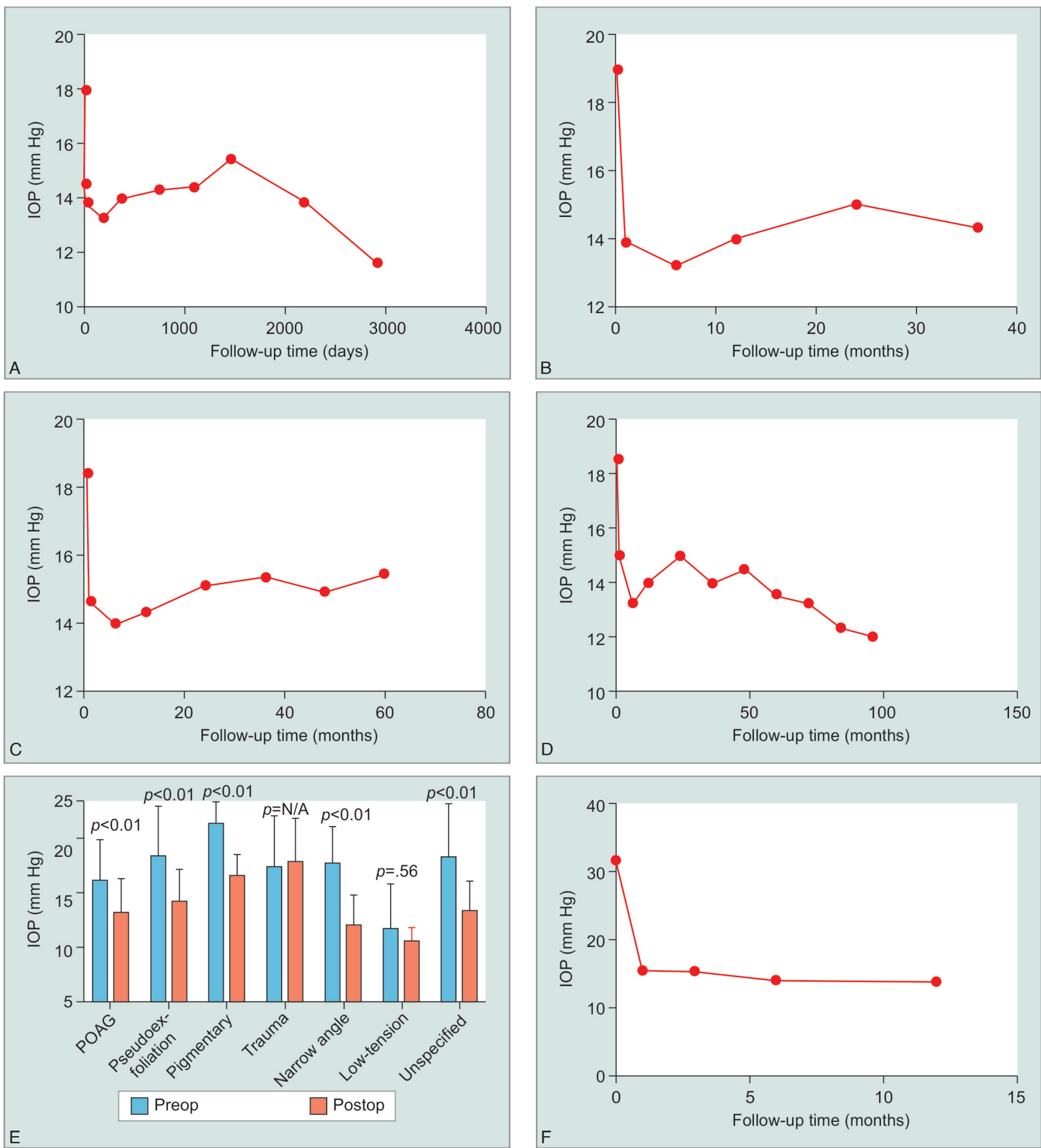

Graphs 1A to F: Intraocular pressure data. (A) Intraocular pressures of all eyes that underwent trabectome procedure at various follow-up times; (B) Intraocular pressures at various time points for patients who had 3 years of follow-up data; (C) Intraocular pressures at various time points for patients who had 5 years of follow-up data; (D) Intraocular pressures at various time points for patients who had 8 years of follow-up data; (E) Intraocular pressures before trabectome and at last follow-up visit after trabectome stratified by type of glaucoma; (F) Intraocular pressures at various time points after trabectome for the eyes that had preoperative IOPs greater than $30 \mathrm{~mm} \mathrm{Hg}$

at final follow-up $(p<0.01)$ (Graphs 1B to D). Graph 1E shows the average reduction of IOP stratified by type of glaucoma. All groups saw a statically significant reduction of IOP at the final follow-up visit, except the lowtension and trauma patients, which had limited follow-up data. Interestingly, we saw the largest decrease in IOP in patients who had been diagnosed with narrow-angle glaucoma. Furthermore, we also explored how effective this procedure was at lowing IOP in refractory cases (preoperative IOP $>30 \mathrm{~mm} \mathrm{Hg}$ ) and saw a mean reduction of $15.53 \mathrm{~mm} \mathrm{Hg}(42.34 \%)$, which was statistically significant $(p<0.01)($ Graph 1F). 
To assess visual outcomes, best corrected visual acuity was expressed on a log MAR scale, and preoperative and postoperative results were compared by type of glaucoma (Graph 2). We found that $68.93 \%$ of eyes saw an improvement of BCVA, $29.30 \%$ saw no significant change and $1.77 \%$ saw a decrease in at the final follow-up visit. The number of preoperative and postoperative glaucoma medications were also compared, and we saw an average decrease from 1.73 medications to 1.13 at the final visit. A paired t-test was used to compare the statistical significance of this finding and a $p$ value of $<0.05$ was determined $(p=0.03)$. Minor complications due to the procedure occurred in $20(5.96 \%)$ of the cases while 0 cases presented significant complications (Table 2). Kaplan-Meier survival analysis was used to determine success based on follow-up time (Graph 3) and showed stable rates up to 100 months postoperatively around about $80 \%$. Of interest, there was no statistical difference in the IOP outcomes after trabectome with or without IOL, including eyes with narrow angles (Graph 4) $(p=0.64)$.

Postoperative pain was one important variable assessed in this study and only $3(0.88 \%)$ of patients reported prolonged issues with pain (Table 2). To determine the success rate of trabectome, patients were split

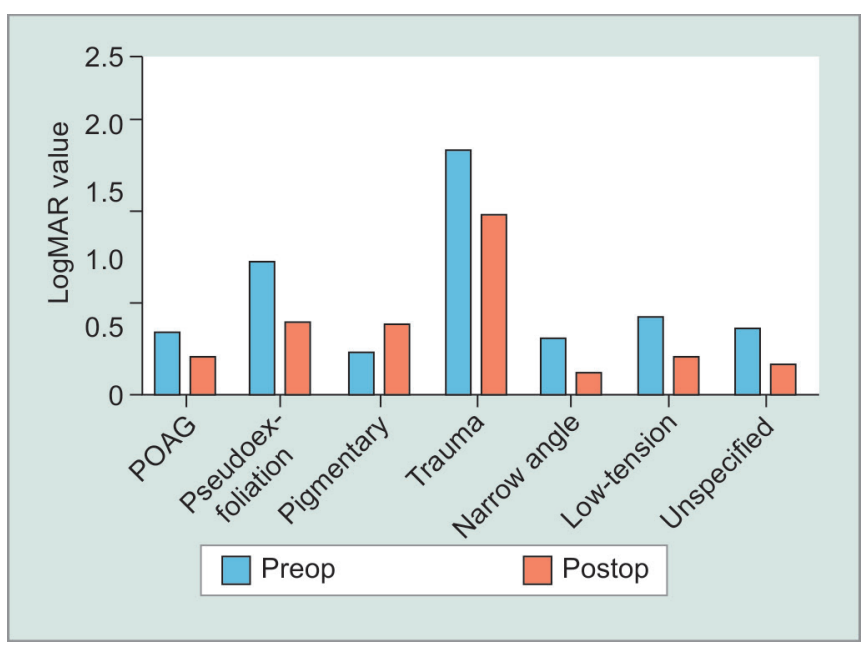

Graph 2: Average LogMAR visual acuity prior to trabectome and at final follow-up visit after trabectome stratified by type of glaucoma

Table 2: Complications and additional procedures required after Trabectome

\begin{tabular}{ll}
\hline Complications & \\
\hline Uncontrolled IOP $(35 \mathrm{~mm} \mathrm{Hg}+)$ & $7(2.06 \%)$ \\
CME & $9(2.65 \%)$ \\
Hyphemia & $16(4.72 \%)$ \\
Conjunctival hemorrhage & $5(1.47 \%)$ \\
Prolonged pain & $3(0.88 \%)$ \\
Additional procedures needed & \\
TSCPC & $2(0.59 \%)$ \\
Valve placement & $3(0.088 \%)$ \\
Laser trabeculoplasty & $1(0.29 \%)$ \\
\hline
\end{tabular}

into two groups based on their average preoperative IOP. Eyes that had a preoperative IOP $>15 \mathrm{~mm} \mathrm{Hg}$ saw success in $85.95 \%$ of cases two years after trabectome while eyes that had a preoperative IOP $<15$ achieved success in $83.99 \%$, giving an overall success rate of $84.96 \%$ two years postop. Kaplan-Meier survival analysis was used to determine success based on follow-up time (Graph 3) and showed stable rates up to 100 months postoperatively of about $80 \%$.

\section{DISCUSSION}

In this study, we evaluated the long-term outcomes of ab-interno trabeculectomy with trabectome to gain insight on how effective this surgery is in stopping or slowing the progression of glaucoma for many years after the operation.

Our cohort of patients, on average, had a lower preoperative IOP than similar studies, ${ }^{6,9,12}$ suggesting that other factors than elevated IOP, such as poor visual acuity and dependence on glaucoma drops, merited surgical intervention. Patients saw an average IOP decrease of $19.58 \%$ at their final visit, which further affirmed the IOP lowering the ability of trabectome highlighted in other studies. ${ }^{9,12-14}$ We also saw a similar average decrease in glaucoma medication at the final follow-up after surgery to many previous studies. ${ }^{9}$ More importantly, we found that in patients who had long term follow-up data, a stable IOP was maintained up to eight years. Of the various types of glaucoma diagnosed, we saw the largest average IOP decrease in eyes with narrow-angle glaucoma. Many surgeons hesitate to perform this type of drainage surgery in narrow-angle patients due to the perceived increased risk, ${ }^{15}$ however Bussel et al. recently showed that there was no correlation of increased risk or decreased effectiveness based on Shaffer grade and our study further affirms this. ${ }^{5}$ In comparing our findings to

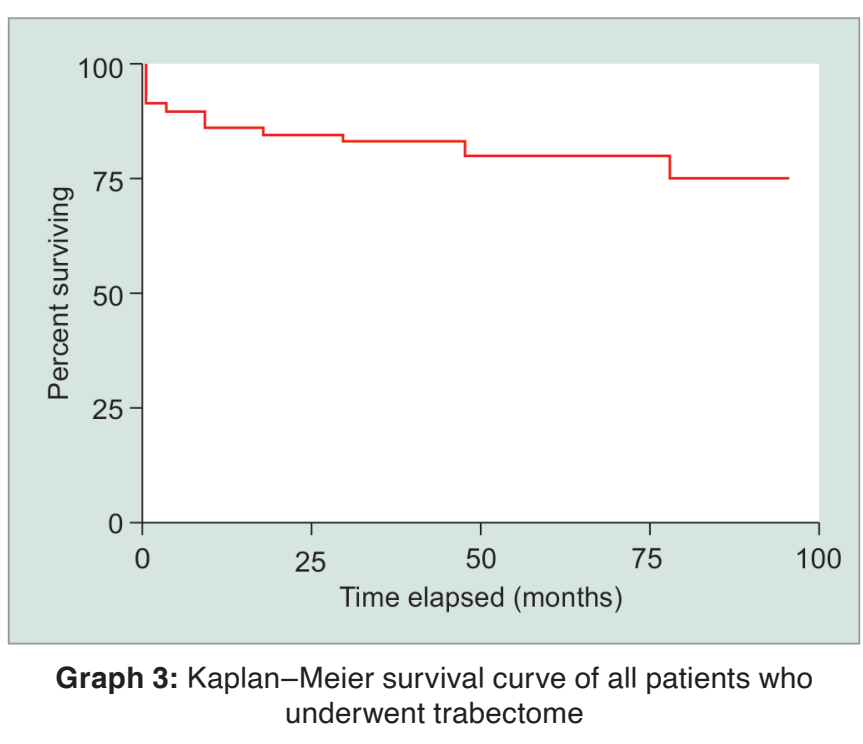



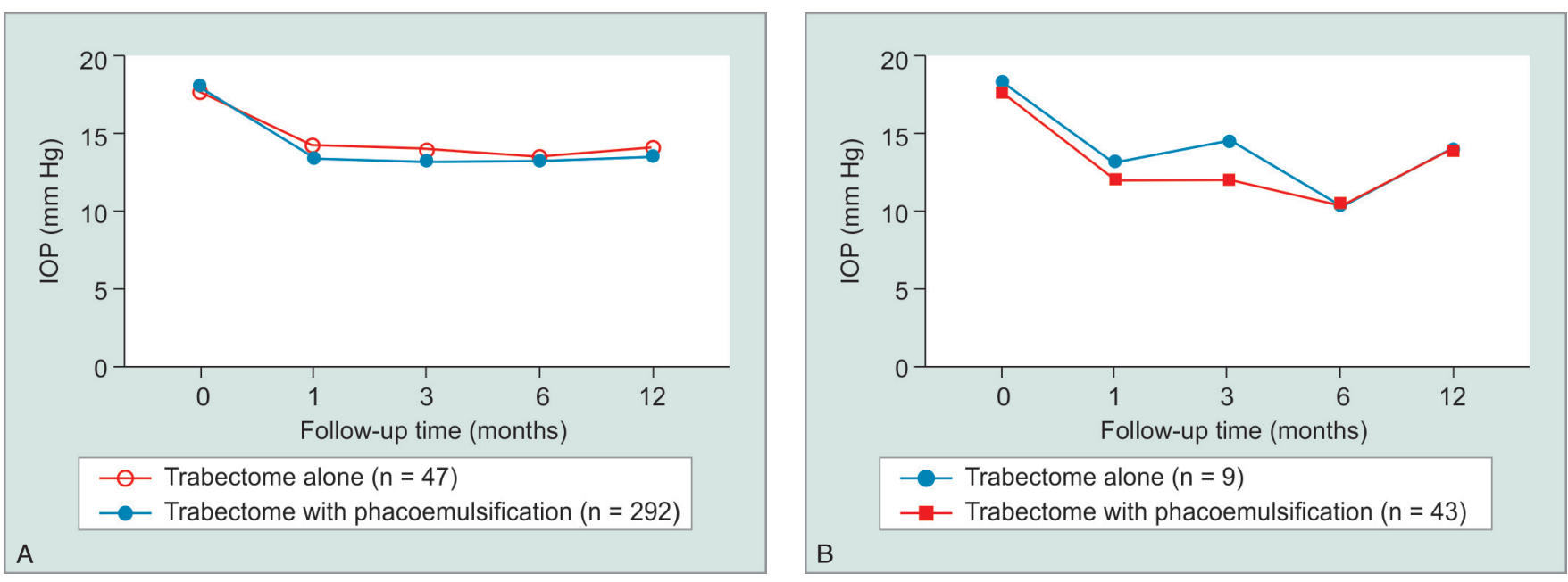

Graphs 4A and B: (A) Intraocular pressures at various follow-up times, comparing eyes that underwent phacoemulsification in conjunction with trabectome vs eyes that received trabectome alone; (B) Intraocular pressures at various follow-up times, comparing eyes that underwent phacoemulsification in conjunction with trabectome vs eyes that received trabectome alone specifically within the angle closure group

similar series, we found few studies that reported outcomes past eighteen months post-operatively. ${ }^{16,17}$ Ahuja et al. published a two-year case study from a cohort of patients who underwent trabectome and found that only $22 \%$ maintained a postoperative IOP $\leq 18 \mathrm{~mm} \mathrm{Hg}$ after two years and suggested that trabectome is only appropriate for patients requiring a target IOP of $21 \mathrm{~mm}$ $\mathrm{Hg}$ or above, ${ }^{17}$ while we found that about $80 \%$ of our patients saw an IOP of $\leq 18 \mathrm{~mm} \mathrm{Hg}$ at two years. Our lower average preoperative IOP could account for the difference in outcomes; however, we believe that our large sample size strongly suggests that the trabectome procedure is successful in maintaining long-term, stable IOPs. Hashemian et al. recently reported in a 30 patients cohort with similar average preoperative IOP to our study, that trabectome was effective at lowering and maintaining IOP 12 months postoperatively..$^{18}$ One limitation of this study was the small sample size; however, we believe that our data further affirms the findings of their study and highlights our conclusion that trabectome is effective even in cases where elevated IOP may not be the main indicator of a need for surgical intervention.

While many studies focus on the IOP lowering ability of IOP with trabectome, ${ }^{5,9,10}$ there is a lack of data on both visual outcomes and overall patient satisfaction. We report that in almost $70 \%$ of cases there was an improvement of visual acuity, while only $1.77 \%$ of eyes saw a substantial decrease in vision. Most cases that saw significant vision loss were rather complex and had undergone multiple prior failed glaucoma procedures. Of the few previous comparable studies done assessing visual outcomes after trabectome, Lee et al. reported in a cohort of Chinese patients with open-angle glaucoma that visual acuity was stable six months postoperatively, ${ }_{1}^{19}$ further confirming our claim.
Regarding long-term patient satisfaction after trabectome, we assessed complication rates, additional surgeries needed and reported prolonged pain. Only 4.17\% required further surgical intervention, which is much lower than previously reported data. $8,14,20$ Of the cases that required additional procedures, over half were due to an inability to maintain a stable IOP. With regards to complication and prolonged reported pain rates, we found that $10.9 \%$ of cases saw minor complications and only $0.88 \%$ of patients reported mild to severe pain at two consecutive visits after trabectome. Taking all this data into consideration suggests that long term patient satisfaction is high.

In comparing our success rates to previous studies on trabectome, we found a one-year success rate of around $85 \%$, which was similar to published data. ${ }^{921-24}$ However, we found much higher success rates compared to the few studies that assessed outcomes over one year. ${ }^{13-14}$ When defining success, we found it useful to divide our cohort into two groups based on their preoperative IOP because it allowed for us to assess outcomes where an elevated IOP was not the main indicator of a need for surgical intervention. Furthermore, when we assessed the IOP lowering ability of trabectome in patients who had a preoperative IOP greater than 30 , we found that in most these cases, multiple previous surgeries were attempted and failed thus giving us insight into trabectome's effect on refractory cases. We found that these patients had a significant decrease in IOP and maintained this up to a year postop. Roy et al. recently reported in a retrospective chart review of 498 cases, that IOP reduction is correlated to glaucoma severity. ${ }^{25}$ We believe that our study is in line with this claim; additionally, we also suggest that trabectome is also effective in less advanced cases. 


\section{CONCLUSION}

Our study suggests that ab-interno trabeculectomy with trabectome is a safe and effective tool for maintaining a stable IOP and visual field up to 8 years. We conclude that trabectome should be considered in a wide variety and severity of glaucoma cases.

\section{CLINICAL SIGNIFICANCE}

While trabectome has been proven to be an effective procedure for lowing IOP in the short term, long term data has yet to be established. Furthermore, minimal data has been reported about the visual outcomes of patients after trabectome. This study provides one of the longest duration of follow up after trabectome surgery published with minimal loss in success out to eight years and great stability of glaucoma control. Our work makes it very clear that regardless of the type or severity of glaucoma, trabectome has an essential place in the surgical options for most glaucoma patients.

\section{REFERENCES}

1. Kingman S. Glaucoma is second leading cause of blindness globally. Bulletin of the World Health Organization. 2004;82:887-888.

2. Resnikoff S, Pascolini D, Etya'Ale D, et al. Global data on visual impairment in the year 2002. Bulletin of the world health organization. 2004;82:844-851.

3. Bussel II, Kaplowitz K, Schuman JS, et al. Outcomes of ab interno trabeculectomy with the trabectome after failed trabeculectomy. British Journal of Ophthalmology. 2015 Feb 1;99(2):258-262.

4. Osman EA, AlMobarak F. Ciliochoroidal effusion with persistent hypotony after trabectome surgery. Indian Journal of Ophthalmology. 2015;63(3):272-274.

5. Bussel II, Kaplowitz K, Schuman JS, et al. Outcomes of ab interno trabeculectomy with the trabectome by degree of angle opening. The British Journal of Ophthalmology. 2015;99(7):914-919.

6. Minckler D, Mosaed S, Dustin L, et al. Trabectome (Trabeculectomy-Internal Approach): Additional Experience and Extended Follow-Up. Transactions of the American Ophthalmological Society. 2008;106:149-160.

7. Brandão LM, Grieshaber MC. Update on Minimally Invasive Glaucoma Surgery (MIGS) and New Implants. Journal of Ophthalmology. 2013;2013:705915.

8. Wecker T, Neuburger M, Bryniok L, et al. Ab Interno Trabeculectomy With the Trabectome as a Valuable Therapeutic Option for Failed Filtering Blebs. J Glaucoma. 2016 Sep;25(9):758-762.

9. Okeke CO, Miller-Ellis E, Rojas M. Trabectome Study Group. Trabectome success factors. Medicine (Baltimore). 2017 Jun;96(24):e7061.
10. Chow JTY, Hutnik CML, Solo K, et al. When Is Evidence Enough Evidence? A Systematic Review and Meta-Analysis of the Trabectome as a Solo Procedure in Patients with Primary Open-Angle Glaucoma. J Ophthalmol. 2017;2017:2965725.

11. Vold SD. Ab interno trabeculotomy with the trabectome system: what does the data tell us? Int Ophthalmol Clin. 2011 Summer;51(3):65-81.

12. Ngai P, Kim G, Chak G, et al. Outcome of primary trabeculotomy ab interno (Trabectome) surgery in patients with steroid-induced glaucoma. Medicine (Baltimore). 2016 Dec;95(50):e5383.

13. Huang $\mathrm{P}$, Wang $\mathrm{H}, \mathrm{Wu} \mathrm{H}$, et al. Preliminary investigation on the safety and efficancy of Trabectome. Dept. of Ophthalmology, Peking Univ. 2015;51(2)115-119.

14. Maeda M, Watanabe M, Ichikawa K. Evaluation of trabectome in open-angle glaucoma. International Journal of Glaucoma. 2013;22:205-208.

15. Liu J, Jung J, Francis BA. Ab interno trabeculotomy: Trabectome TM surgical treatment for open-angle glaucoma. Expert Rev Ophthalmol 2009;4:119-128.

16. Mizoguchi T, Nishigaki S, Sato T, et al. Clinical results of Trabectome surgery for open-angle glaucoma. Clin Ophthalmol. 2015 Oct 9;9:1889-1894.

17. Ahuja $\mathrm{Y}, \mathrm{Ma}$ Khin Pyi S, Malihi M, Hodge DO, Sit AJ. Clinical results of ab interno trabeculotomy using the trabectome for open-angle glaucoma: the Mayo Clinic series in Rochester, Minnesota. Am J Ophthalmol. 2013 Nov;156(5): 927-935.e2.

18. Hashemian SJ, Miraftabi A, Jafari ME, et al. Combined cataract extraction and trabeculotomy by the internal approach for coexisting cataract and open-angle glaucoma. J Curr Ophthalmol. 2016 Oct 4;29(1):17-22.

19. Lee JW, Yick DW, Tsang S, et al. Efficacy and Safety of Trabectome Surgery in Chinese Open-Angle Glaucoma. Medicine (Baltimore). 2016 Apr;95(15):e3212.

20. Shoji N, Kasahara M, Iijima A, et al. Short-term evaluation of Trabectome surgery performed on Japanese patients with open-angle glaucoma. Jpn J Ophthalmol. 2016 May;60(3):156-165.

21. Loewen RT, Roy P, Parikh HA, et al. Impact of a Glaucoma Severity Index on Results of Trabectome Surgery: Larger Pressure Reduction in More Severe Glaucoma. PLoS One. 2016 Mar 23;11(3):e0151926.

22. Yildirim Y, Kar T, Duzgun E, et al. Evaluation of the longterm results of trabectome surgery. Int Ophthalmol. 2016 Oct;36(5):719-726.

23. Werth JP, Gesser C, Klemm M. Diverse effectiveness of the trabectome for different types of glaucoma. Klin Monbl Augenheilkd. 2015 Jan;232(1):72-78.

24. Ting JL, Damji KF, Stiles MC. Trabectome Study Group. $\mathrm{Ab}$ interno trabeculectomy: outcomes in exfoliation versus primary open-angle glaucoma. J Cataract Refract Surg. 2012 Feb;38(2):315-323.

25. Roy P, Loewen RT, Dang Y, et al. Stratification of phacotrabectome surgery results using a glaucoma severity index in a retrospective analysis. BMC Ophthalmol. 2017 Mar 21;17(1):30. 